Research Article

\title{
On the Starlikeness of Certain Class of Multivalent Analytic Functions
}

\author{
Lei Shi and Zhi-Gang Wang \\ School of Mathematics and Statistics, Anyang Normal University, Anyang, Henan 455000, China \\ Correspondence should be addressed to Zhi-Gang Wang; zhigangwang@foxmail.com
}

Received 22 October 2013; Revised 5 March 2014; Accepted 5 March 2014; Published 30 March 2014

Academic Editor: Pavel Kurasov

Copyright (C) 2014 L. Shi and Z.-G. Wang. This is an open access article distributed under the Creative Commons Attribution License, which permits unrestricted use, distribution, and reproduction in any medium, provided the original work is properly cited.

The main purpose of this paper is to determine the conditions of starlikeness for certain class of multivalent analytic functions. Relevant connections of the results presented here with those obtained in earlier works are pointed out.

\section{Introduction and Main Result}

Let $\mathscr{A}_{p}$ denote the class of functions of the form

$$
f(z)=z^{p}+\sum_{n=p+1}^{\infty} a_{n} z^{n} \quad(p \in \mathbb{N}:=\{1,2, \ldots\}),
$$

which are analytic in the open unit disk $\mathbb{U}:=\{z: z \in$ $\mathbb{C}$ and $|z|<1\}$. For convenience, we set $\mathscr{A}_{1}=: \mathscr{A}$. A function $f \in \mathscr{A}_{p}$ is said to be in the class $\mathcal{S}_{p}^{*}(\varrho)$ of $p$-valent starlike functions of order $\varrho$ in $\mathbb{U}$, if it satisfies the following inequality:

$$
\mathfrak{R}\left(\frac{z f^{\prime}(z)}{f(z)}\right)>\varrho \quad(0 \leqq \varrho<p ; z \in \mathbb{U}) .
$$

For simplicity, we write $\mathcal{S}_{1}^{*}(0)=: \mathcal{S}^{*}$.

In [1], Chichra introduced the class $\mathscr{R}$ of analytic functions $f \in \mathscr{A}$ which satisfy the condition

$$
\mathfrak{R}\left(f^{\prime}(z)+z f^{\prime \prime}(z)\right)>0 \quad(z \in \mathbb{U}) .
$$

He proved that the members of $\mathscr{R}$ are univalent in $\mathbb{U}$. Later, R. Singh and S. Singh [2] showed that $\mathscr{R} \subset \mathcal{S}^{*}$. Recently, Gao and Zhou [3] considered the subclass $\mathscr{R}(\beta, \gamma)$ of $\mathscr{A}$ which is defined by

$$
\begin{array}{r}
\mathscr{R}(\beta, \gamma):=\left\{f \in \mathscr{A}: \mathfrak{R}\left(f^{\prime}(z)+\beta z f^{\prime \prime}(z)\right)>\gamma\right\} \\
(\beta>0 ; \gamma<1 ; z \in \mathbb{U}) .
\end{array}
$$

They derived some mapping properties of this class. Moreover, several authors discussed some related analytic function classes associated with the class $\mathscr{R}$ (see [4-7]). By using the method of differential subordination, Yang and Liu [8] generalized the above works and studied the subclass $\mathscr{T}_{p}(A, B, \gamma, \alpha)$ of $\mathscr{A}_{p}$ which satisfies the condition

$$
f^{\prime}(z)+\alpha z f^{\prime \prime}(z) \prec h(z) \quad(z \in \mathbb{U}),
$$

where

$$
h(z)= \begin{cases}\left(\frac{1+A z}{1+B z}\right)^{\gamma}, & (A \leqq 1 ; 0<\gamma<1), \\ \frac{1+A z}{1+B z}, & (\gamma=1) .\end{cases}
$$

In [9], Owa et al. introduced a new subclass $\mathscr{R}_{p}(\alpha, \beta, \gamma ; j)$ of $\mathscr{A}_{p}$ which satisfies the inequality

$$
\Re\left(\alpha \frac{f^{(j)}(z)}{z^{p^{-j}}}+\beta \frac{f^{(j+1)}(z)}{z^{p^{-j-1}}}\right)>\gamma
$$

$$
(j \in\{0,1,2, \ldots, p\} ; p \in \mathbb{N} ; z \in \mathbb{U}),
$$


where $\alpha>0, \beta>0$, and (throughout this paper unless otherwise mentioned) the parameters $\gamma$ and $\delta$ are constrained as follows:

$$
\begin{gathered}
\gamma<\delta:=\frac{p ![\alpha+(p-j) \beta]}{(p-j) !} \\
(\alpha>0 ; \beta>0 ; p \in \mathbb{N} ; j \in\{0,1,2, \ldots, p\}) .
\end{gathered}
$$

The extreme points, coefficient inequalities, radius of starlikeness, and inclusion relationship for the class $\mathscr{R}_{p}(\alpha, \beta, \gamma ; j)$ are derived. By setting $p=j=\alpha=1$, it is easy to see that the class $\mathscr{R}_{p}(\alpha, \beta, \gamma ; j)$ reduces to the class $\mathscr{R}(\beta, \gamma)$. If we set $p=j=\alpha=\gamma=1$ in the class $\mathscr{R}_{p}(\alpha, \beta, \gamma ; j)$, then it reduces to the class $\mathscr{R}(\gamma)$, which was studied earlier by Silverman [10], R. Singh and S. Singh $[2,11]$, independently.

For some recent investigations on the starlikeness of analytic functions, one can refer to [12-20]. In the present paper, we aim at deriving the conditions of starlikeness for the class $\mathscr{R}_{p}(\alpha, \beta, \gamma ; j)$. The main result is presented below.

Theorem 1. Let $\beta \geqq \alpha>0$. Then

(1) $\mathscr{R}_{p}(\alpha, \beta, \gamma ; 1) \subset \mathcal{S}_{p}^{*}$ for $\gamma_{1} \leqq \gamma<p[\alpha+(p-1) \beta]$, where $\gamma_{1}$ is the solution of the following equation:

$$
\begin{aligned}
& -\frac{p}{2}\left(1+2\left\{p[\alpha+(p-1) \beta]-\gamma_{1}\right\}\right. \\
& \left.\quad \times \sum_{n=2}^{\infty} \frac{(-1)^{n-1}}{(n+p-1)[\alpha+(n+p-2) \beta]}\right) \\
& =\frac{\gamma_{1}}{\beta}+\frac{\beta-\alpha}{\beta} \\
& \times\left(p+2\left\{p[\alpha+(p-1) \beta]-\gamma_{1}\right\}\right. \\
& \left.\quad \times \sum_{n=2}^{\infty} \frac{(-1)^{n-1}}{\alpha+(n+p-2) \beta}\right) ;
\end{aligned}
$$

(2) $\mathscr{R}_{p}(\alpha, \beta, \gamma ; j) \subset \mathcal{S}_{p}^{*}(j-1)(j \in\{2,3, \ldots, p\})$ for $\gamma_{2} \leqq$ $\gamma<p[\alpha+(p-1) \beta]$, where $\gamma_{2}$ is the solution of the following equation:

$$
\begin{aligned}
& -\frac{p-j+1}{2}\left(\frac{p !}{(p-j+1) !}+2\left(\delta-\gamma_{2}\right)\right. \\
& \left.\quad \times \sum_{n=2}^{\infty} \frac{(-1)^{n-1}}{\alpha+(n+p-j) \beta}\right) \\
& =\frac{\gamma_{2}}{\beta}+\frac{\beta-\alpha}{\beta} \\
& \times\left(\frac{p !}{(p-j) !}+2\left(\delta-\gamma_{2}\right)\right. \\
& \left.\quad \times \sum_{n=2}^{\infty} \frac{(-1)^{n-1}}{\alpha+(n+p-j-1) \beta}\right) .
\end{aligned}
$$

\section{Preliminary Results}

In order to establish our main theorem, we will require the following lemmas.

Lemma 2 (see [9]). A function $f \in \mathscr{R}_{p}(\alpha, \beta, \gamma ; j)$ if and only if $f$ can be expressed as follows:

$$
\begin{aligned}
f(z)= & z^{p}+2(\delta-\gamma) \\
& \times \int_{|x|=1}\left(\sum_{n=p+1}^{\infty} \frac{(n-j) !}{n ![\alpha+(n-j) \beta]} x^{n-p} z^{n}\right) d \mu(x),
\end{aligned}
$$

where $\mu(x)$ is the probability measure on $\mathbb{X}:=\{x \in \mathbb{C}:|x|=$ $1\}$.

The proof of the following lemma is much akin to that of Theorem 1 which was obtained by Nunokawa et al. [21] (see also Liu [22] and Yang [23]). We, therefore, choose to omit the analogous details involved.

Lemma 3. If $f \in \mathscr{A}_{p}$ satisfies the inequality

$$
\mathfrak{R}\left(\frac{z f^{(j)}(z)}{f^{(j-1)}(z)}\right)>0 \quad(j \in\{1,2, \ldots, p\} ; z \in \mathbb{U}),
$$

then $f \in \mathcal{S}_{p}^{*}(j-1)$.

Lemma 4 (Jack's Lemma [24]). Let $\phi$ be a nonconstant regular function in $\mathbb{U}$. If $|\phi|$ attains its maximum value on the circle $|z|=r<1$ at $z_{0}$, then

$$
z_{0} \phi^{\prime}\left(z_{0}\right)=k \phi\left(z_{0}\right)
$$

where $k \geqq 1$ is a real number.

We now give the lower bounds of the following continuous linear operators:

$$
\begin{gathered}
\mathscr{L}_{1}(f)=\mathfrak{R}\left(\frac{f(z)}{z^{p}}\right) \quad\left(f \in \mathscr{R}_{p}(\alpha, \beta, \gamma ; 1) ; z \in \mathbb{U}\right), \\
\mathscr{L}_{2}(f)=\mathfrak{R}\left(\frac{f^{(j)}(z)}{z^{p-j}}\right) \quad\left(f \in \mathscr{R}_{p}(\alpha, \beta, \gamma ; j) ;\right. \\
j \in\{1,2, \ldots, p\} ; z \in \mathbb{U})
\end{gathered}
$$

acting on the class $\mathscr{R}_{p}(\alpha, \beta, \gamma ; j)$, which played crucial role in the proof of our main result.

Lemma 5. If $f \in \mathscr{R}_{p}(\alpha, \beta, \gamma ; j)$, then, for $|z| \leqq r<1$, one has the following. 
(1) When $j \in\{1,2, \ldots, p\}$, then

$$
\begin{aligned}
\Re\left(\frac{f^{(j)}(z)}{z^{p-j}}\right) \geqq & \frac{p !}{(p-j) !} \\
& +2(\delta-\gamma) \sum_{n=2}^{\infty} \frac{(-r)^{n-1}}{\alpha+(n+p-j-1) \beta} \\
> & \frac{p !}{(p-j) !} \\
& +2(\delta-\gamma) \sum_{n=2}^{\infty} \frac{(-1)^{n-1}}{\alpha+(n+p-j-1) \beta} .
\end{aligned}
$$

This inequality is sharp.

(2) When $j=1$, then

$$
\begin{aligned}
& \Re\left(\frac{f(z)}{z^{p}}\right) \\
& \quad \geqq 1+2\left(\delta_{1}-\gamma\right) \sum_{n=2}^{\infty} \frac{(-r)^{n-1}}{(n+p-1)[\alpha+(n+p-2) \beta]} \\
& \quad>1+2\left(\delta_{1}-\gamma\right) \sum_{n=2}^{\infty} \frac{(-1)^{n-1}}{(n+p-1)[\alpha+(n+p-2) \beta]}
\end{aligned}
$$

where $\delta_{1}=p[\alpha+(p-1) \beta]$. The inequality is sharp.

Proof. By Lemma 2, we know that

$$
f(z)=z^{p}+2(\delta-\gamma) \sum_{n=p+1}^{\infty} \frac{(n-j) !}{n ![\alpha+(n-j) \beta]} z^{n}
$$

is the extreme function of the class $\mathscr{R}_{p}(\alpha, \beta, \gamma ; j)$. Thus, we only need to consider the function $f$ defined by (17); it follows that

$$
\frac{f^{(j)}(z)}{z^{p-j}}=\frac{p !}{(p-j) !}+2(\delta-\gamma) \sum_{n=2}^{\infty} \frac{(-r)^{n-1}}{\alpha+(n+p-j-1) \beta} z^{n-1}
$$

We note that (18) can be written as follows:

$$
\frac{f^{(j)}(z)}{z^{p^{-j}}}=\frac{p !}{(p-j) !}+\frac{2(\delta-\gamma)}{\beta} \int_{0}^{1} t^{(\alpha / \beta)+p-j} \frac{z}{1-t z} d t .
$$

Thus, we find from (19) that

$$
\begin{aligned}
\Re\left(\frac{f^{(j)}(z)}{z^{p-j}}\right)= & \frac{p !}{(p-j) !}+\frac{2(\delta-\gamma)}{\beta} \\
& \times \int_{0}^{1} t^{(\alpha / \beta)+p^{-j}} \mathfrak{R}\left(\frac{z}{1-t z}\right) d t .
\end{aligned}
$$

Moreover, we observe that the function

$$
k(z)=\frac{z}{1-t z} \quad(0 \leqq t \leqq 1)
$$

is convex in $\mathbb{U}, k(\bar{z})=\overline{k(z)}$, and $k(z)$ maps real axis to real axis; we have

$$
-\frac{r}{1+t r} \leqq \mathfrak{R}\left(\frac{z}{1-t z}\right) \leqq \frac{r}{1-t r} \quad(|z| \leqq r<1) .
$$

Upon substituting (22) into (20) and expanding the integrand into the power series of $t$ and integrating it, we can easily get (15). The sharpness of (15) can be seen from (18).

By similarly applying the method of proof of (15), we also can prove (16) holds true. The sharpness of (16) can be found in (17).

\section{Proof of Theorem 1}

Proof. Suppose that $f \in \mathscr{R}_{p}(\alpha, \beta, \gamma ; j)$ with $\beta \geqq \alpha>0$. It follows from (7) that

$$
\mathfrak{R}\left(\frac{f^{(j)}(z)}{z^{p-j}}+\frac{\beta}{\alpha} \frac{f^{(j+1)}(z)}{z^{p-j-1}}\right)>\frac{\gamma}{\alpha} \quad(z \in \mathbb{U}) .
$$

By noting that

$$
\begin{aligned}
& \frac{\beta}{\alpha}\left(\frac{f^{(j)}(z)}{z^{p^{-j}}}+\frac{f^{(j+1)}(z)}{z^{p-j-1}}\right) \\
& \quad=\frac{f^{(j)}(z)}{z^{p-j}}+\frac{\beta}{\alpha} \frac{f^{(j+1)}(z)}{z^{p-j-1}}+\left(\frac{\beta}{\alpha}-1\right) \frac{f^{(j)}(z)}{z^{p-j}},
\end{aligned}
$$

we get

$$
\begin{aligned}
\mathfrak{R}\left(\frac{f^{(j)}(z)}{z^{p-j}}+\frac{f^{(j+1)}(z)}{z^{p-j-1}}\right)= & \frac{\alpha}{\beta} \mathfrak{R}\left(\frac{f^{(j)}(z)}{z^{p-j}}+\frac{\beta}{\alpha} \frac{f^{(j+1)}(z)}{z^{p-j-1}}\right) \\
& +\frac{\beta-\alpha}{\beta} \mathfrak{R}\left(\frac{f^{(j)}(z)}{z^{p-j}}\right) .
\end{aligned}
$$

Thus, we can easily find from (15), (23), and (25) that

$$
\begin{aligned}
& \Re\left(\frac{f^{(j)}(z)}{z^{p-j}}+\frac{f^{(j+1)}(z)}{z^{p-j-1}}\right) \\
& >\frac{\gamma}{\beta}+\frac{\beta-\alpha}{\beta} \\
& \quad \times\left(\frac{p !}{(p-j) !}+2(\delta-\gamma) \sum_{n=2}^{\infty} \frac{(-1)^{n-1}}{\alpha+(n+p-j-1) \beta}\right) .
\end{aligned}
$$

We now set

$$
\begin{array}{r}
\frac{f^{(j)}(z)}{f^{(j-1)}(z)}=(p-j+1) \frac{1+\omega(z)}{1-\omega(z)} \\
(j \in\{1,2, \ldots, p\} ; z \in \mathbb{U}) .
\end{array}
$$


Then $\omega$ is analytic in $\mathbb{U}$ with $\omega(0)=0$. It follows from (27) that

$$
\begin{aligned}
& \frac{f^{(j)}(z)}{z^{p-j}}+\frac{f^{(j+1)}(z)}{z^{p-j-1}} \\
& =(p-j+1) \frac{f^{(j-1)}(z)}{z^{p-j+1}}\left[\left(\frac{1+\omega(z)}{1-\omega(z)}\right)^{2}+\frac{2 z \omega^{\prime}(z)}{(1-\omega(z))^{2}}\right] .
\end{aligned}
$$

At the same time, we can claim that $|\omega(z)|<1$. Indeed, if not, there exists a point $z_{0} \in \mathbb{U}$ such that

$$
\max _{|z| \leqq\left|z_{0}\right|}|\omega(z)|=\left|\omega\left(z_{0}\right)\right|=1
$$

by Lemma 4, we obtain

$$
z_{0} \omega^{\prime}\left(z_{0}\right)=k \omega\left(z_{0}\right)=k e^{i \theta} \quad(0<\theta<2 \pi ; k \geqq 1) .
$$

For $z=z_{0}$, by virtue of (28), we split it into two cases to prove the following.

(1) When $j=1$, in view of (16), we get

$$
\begin{aligned}
& \Re\left(\frac{f^{\prime}\left(z_{0}\right)}{z_{0}^{p-1}}+\frac{f^{\prime \prime}\left(z_{0}\right)}{z_{0}^{p-2}}\right) \\
& =p \Re\left(\frac{f\left(z_{0}\right)}{z_{0}^{p}}\left[\left(\frac{1+e^{i \theta}}{1-e^{i \theta}}\right)^{2}+\frac{2 k e^{i \theta}}{\left(1-e^{i \theta}\right)^{2}}\right]\right) \\
& \leqq-\frac{p k}{2 \sin ^{2}(\theta / 2)} \mathfrak{R}\left(\frac{f\left(z_{0}\right)}{z_{0}^{p}}\right) \leqq-\frac{p}{2} \mathfrak{R}\left(\frac{f\left(z_{0}\right)}{z_{0}^{p}}\right) \\
& \leqq-\frac{p}{2}(1+2\{p[\alpha+(p-1) \beta]-\gamma\} \\
& \left.\quad \times \sum_{n=2}^{\infty} \frac{(-1)^{n-1}}{(n+p-1)[\alpha+(n+p-2) \beta]}\right) .
\end{aligned}
$$

Let $\beta \geqq \alpha>0$. If $\gamma$ satisfies the condition

$$
\begin{aligned}
& -\frac{p}{2}(1+2\{p[\alpha+(p-1) \beta]-\gamma\} \\
& \left.\quad \times \sum_{n=2}^{\infty} \frac{(-1)^{n-1}}{(n+p-1)[\alpha+(n+p-2) \beta]}\right) \\
& \leqq \frac{\gamma}{\beta}+\frac{\beta-\alpha}{\beta} \\
& \quad \times(p+2\{p[\alpha+(p-1) \beta]-\gamma\} \\
& \left.\quad \times \sum_{n=2}^{\infty} \frac{(-1)^{n-1}}{\alpha+(n+p-j-1) \beta}\right),
\end{aligned}
$$

we have a contradiction to (26) at $z=z_{0}$; the smallest $\gamma$ satisfies (32) is solution $\gamma_{1}$ of (9). This implies that if $\beta \geqq \alpha>0$ and $\gamma_{1} \leqq \gamma<p[\alpha+(p-1) \beta]$, we have $|\omega(z)|<1$. Thus, we conclude that $f \in \mathcal{S}_{p}^{*}$.
(2) When $j \in\{2,3, \ldots, p\}$, by means of (15), we have

$$
\begin{aligned}
& \Re\left(\frac{f^{(j)}\left(z_{0}\right)}{z_{0}^{p-j}}+\frac{f^{(j+1)}\left(z_{0}\right)}{z_{0}^{p-j-1}}\right) \\
&=(p-j+1) \Re\left(\frac{f^{(j-1)}\left(z_{0}\right)}{z_{0}^{p-j+1}}\left[\left(\frac{1+e^{i \theta}}{1-e^{i \theta}}\right)^{2}+\frac{2 k e^{i \theta}}{\left(1-e^{i \theta}\right)^{2}}\right]\right) \\
& \leqq-\frac{(p-j+1) k}{2 \sin ^{2}(\theta / 2)} \mathfrak{R}\left(\frac{f^{(j-1)}\left(z_{0}\right)}{z_{0}^{p-j+1}}\right) \\
& \leqq-\frac{p-j+1}{2} \mathfrak{R}\left(\frac{f^{(j-1)}\left(z_{0}\right)}{z_{0}^{p-j+1}}\right) \\
& \leqq \frac{p-j+1}{2} \\
& \times\left(\frac{p !}{(p-j+1) !}+2(\delta-\gamma) \sum_{n=2}^{\infty} \frac{(-1)^{n-1}}{\alpha+(n+p-j) \beta}\right) .
\end{aligned}
$$

Let $\beta \geqq \alpha>0$. If $\gamma$ satisfies the following inequality,

$$
\begin{aligned}
- & \frac{p-j+1}{2} \\
& \times\left(\frac{p !}{(p-j+1) !}+2(\delta-\gamma) \sum_{n=2}^{\infty} \frac{(-1)^{n-1}}{\alpha+(n+p-j) \beta}\right) \\
\leqq & \frac{\gamma}{\beta}+\frac{\beta-\alpha}{\beta} \\
& \times\left(\frac{p !}{(p-j) !}+2(\delta-\gamma) \sum_{n=2}^{\infty} \frac{(-1)^{n-1}}{\alpha+(n+p-j-1) \beta}\right),
\end{aligned}
$$

we have a contradiction to (26) at $z=z_{0}$; the smallest $\gamma$ satisfies (34) is solution $\gamma_{2}$ of (10). This shows that if $\beta \geqq \alpha>0$ and $\gamma_{2} \leqq \gamma<\delta$, we have $|\omega(z)|<1$. It follows from (27) that

$$
\mathfrak{R}\left(\frac{z f^{(j)}(z)}{f^{(j-1)}(z)}\right)>0 \quad(j \in\{2,3, \ldots, p\} ; z \in \mathbb{U}) .
$$

Therefore, by Lemma 3, we deduce that

$$
\mathscr{R}_{p}(\alpha, \beta, \gamma ; j) \in \mathcal{S}_{p}^{*}(j-1) \quad(j \in\{2,3, \ldots, p\}) .
$$

The proof of the theorem is thus completed.

Remark 6. If $\beta<\alpha(\alpha>0 ; \beta>0)$, we cannot find the number $\gamma(\alpha, \beta)$ such that

$$
\mathscr{R}_{p}(\alpha, \beta, \gamma ; j) \in \mathcal{S}_{p}^{*}(j-1) \quad(j \in\{2,3, \ldots, p\}),
$$

since the continuous linear operators $\mathscr{L}_{1}(f)$ and $\mathscr{L}_{2}(f)$ acting on $\mathscr{R}_{p}(\alpha, \beta, \gamma ; j)$ do not exist in sharp upper bounds.

Putting $p=\alpha=1$ in the first part of Theorem 1, we can get the following result. 
Corollary 7. Let $\beta \geqq 1$. Then $\mathscr{R}(\beta, \gamma) \subset \mathcal{S}^{*}$ for $\gamma_{3} \leqq \gamma<1$, where $\gamma_{3}$ is the solution of the following equation:

$$
1-\frac{3}{2} \beta=\gamma_{3}+\left(1-\gamma_{3}\right) \sum_{n=2}^{\infty} \frac{(-1)^{n-1}[\beta+2 n(\beta-1)]}{n[1+(n-1) \beta]} .
$$

Remark 8. Corollary 7 corrects some errors of Theorem 4 in [3].

By setting $p=\alpha=\beta=1$ in the first part of Theorem 1 , we also can get the following criterion for starlikeness obtained by Silverman [10].

Corollary 9. Consider $\mathscr{R}(\gamma) \subset \mathcal{S}^{*}$ for $\left(6-\pi^{2}\right) /\left(24-\pi^{2}\right) \leqq$ $\gamma<1$.

\section{Conflict of Interests}

The authors declare that there is no conflict of interests regarding the publication of this paper.

\section{Acknowledgments}

The present investigation was supported by the National Natural Science Foundation under Grant nos. 11301008, 11226088, and 11101053, the Foundation for Excellent Youth Teachers of Colleges and Universities of Henan Province under Grant no. 2013GGJS-146, and the Natural Science Foundation of Educational Committee of Henan Province under Grant 14B110012 of China. The authors are grateful to the referees for their valuable comments and suggestions which essentially improved the quality of this paper.

\section{References}

[1] P. N. Chichra, "New subclasses of the class of close-to-convex functions," Proceedings of the American Mathematical Society, vol. 62 , no. 1, pp. 37-43, 1977.

[2] R. Singh and S. Singh, "Starlikeness and convexity of certain integrals," Annales Universitatis Mariae Curie-Skłodowska A, vol. 35, pp. 45-47, 1981.

[3] C.-Y. Gao and S.-Q. Zhou, "Certain subclass of starlike functions," Applied Mathematics and Computation, vol. 187, no. 1, pp. 176-182, 2007.

[4] Y. C. Kim, "Mapping properties of differential inequalities related to univalent functions," Applied Mathematics and Computation, vol. 187, no. 1, pp. 272-279, 2007.

[5] Y. C. Kim and H. M. Srivastava, "Some applications of a differential subordination," International Journal of Mathematics and Mathematical Sciences, vol. 22, no. 3, pp. 649-654, 1999.

[6] H. M. Srivastava, N.-E. Xu, and D.-G. Yang, "Inclusion relations and convolution properties of a certain class of analytic functions associated with the Ruscheweyh derivatives," Journal of Mathematical Analysis and Applications, vol. 331, no. 1, pp. 686700, 2007.

[7] D.-G. Yang and J.-L. Liu, "On a class of analytic functions with missing coefficients," Applied Mathematics and Computation, vol. 215, no. 9, pp. 3473-3481, 2010.

[8] D.-G. Yang and J.-L. Liu, "A class of analytic functions with missing coefficients," Abstract and Applied Analysis, vol. 2011, Article ID 456729, 16 pages, 2011.
[9] S. Owa, T. Hayami, and K. Kuroki, "Some properties of certain analytic functions," International Journal of Mathematics and Mathematical Sciences, vol. 2007, Article ID 91592, 9 pages, 2007.

[10] H. Silverman, "A class of bounded starlike functions," International Journal of Mathematics and Mathematical Sciences, vol. 17, no. 2, pp. 249-252, 1994.

[11] R. Singh and S. Singh, "Convolution properties of a class of starlike functions," Proceedings of the American Mathematical Society, vol. 106, no. 1, pp. 145-152, 1989.

[12] M. Nunokawa and J. Sokół, "An improvement of Ozaki’s condition," Applied Mathematics and Computation, vol. 219, no. 22, pp. 10768-10776, 2013.

[13] M. Nunokawa and J. Sokół, "Remarks on some starlike functions," Journal of Inequalities and Applications, vol. 2013, article 593, pp. 1-8, 2013.

[14] S. Siregar, "The starlikeness of analytic functions of Koebe type," Mathematical and Computer Modelling, vol. 54, no. 11-12, pp. 2928-2938, 2011.

[15] S. Sivasubramanian, M. Darus, and R. W. Ibrahim, "On the starlikeness of certain class of analytic functions," Mathematical and Computer Modelling, vol. 54, no. 1-2, pp. 112-118, 2011.

[16] J. Sokół and M. Nunokawa, "On some sufficient conditions for univalence and starlikeness," Journal of Inequalities and Applications, vol. 2012, article 282, pp. 1-11, 2012.

[17] H. M. Srivastava and A. Y. Lashin, "Subordination properties of certain classes of multivalently analytic functions," Mathematical and Computer Modelling, vol. 52, no. 3-4, pp. 596-602, 2010.

[18] Z.-G. Wang, Z.-H. Liu, and R.-G. Xiang, "Some criteria for meromorphic multivalent starlike functions," Applied Mathematics and Computation, vol. 218, no. 3, pp. 1107-1111, 2011.

[19] Z.-G. Wang, Y. Sun, and N. Xu, "Some properties of certain meromorphic close-to-convex functions," Applied Mathematics Letters, vol. 25, no. 3, pp. 454-460, 2012.

[20] Z.-G. Wang, H. M. Srivastava, and S-M. Yuan, "Some basic properties of certain subclasses of meromorphically starlike functions," Journal of Inequalities and Applications, vol. 2014, article 29, pp. 1-13, 2014.

[21] M. Nunokawa, S. Owa, T. Sekine, R. Yamakawa, H. Saitoh, and J. Nishiwaki, "On certain multivalent functions," International Journal of Mathematics and Mathematical Sciences, vol. 2007, Article ID 72393, 5 pages, 2007.

[22] J.-L. Liu, "Remark on two results of Nunokawa," Journal of Mathematical Research and Exposition, vol. 16, no. 3, pp. 351354, 1996.

[23] D.-G. Yang, "Some criteria for $p$-valent functions," Bulletin of the Korean Mathematical Society, vol. 35, no. 3, pp. 571-582, 1998.

[24] I. S. Jack, "Functions starlike and convex of order $\alpha$," Journal of the London Mathematical Society, vol. 3, pp. 469-474, 1971. 


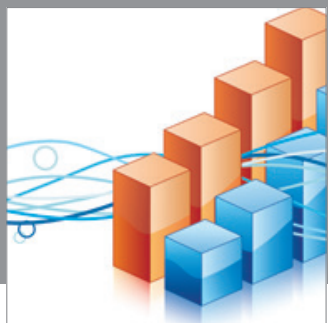

Advances in

Operations Research

mansans

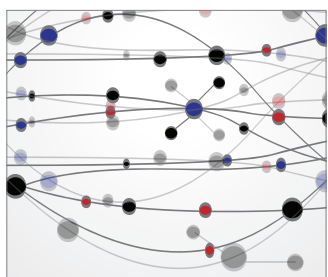

The Scientific World Journal
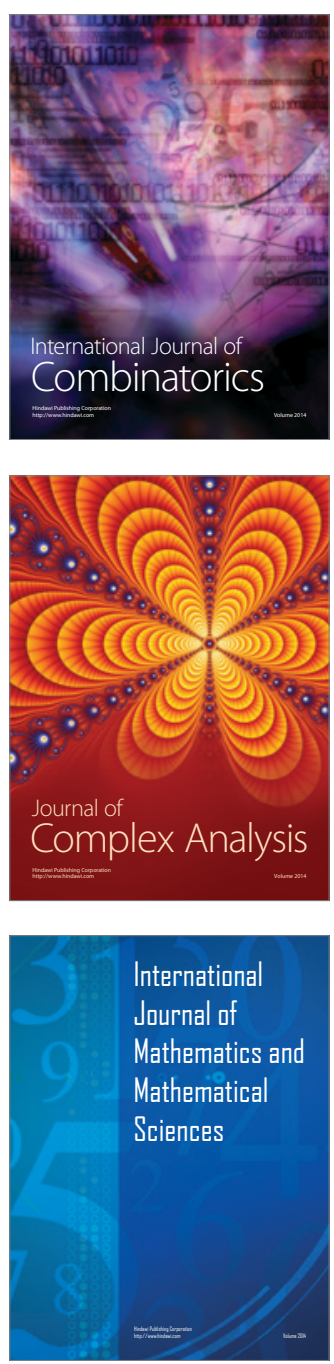
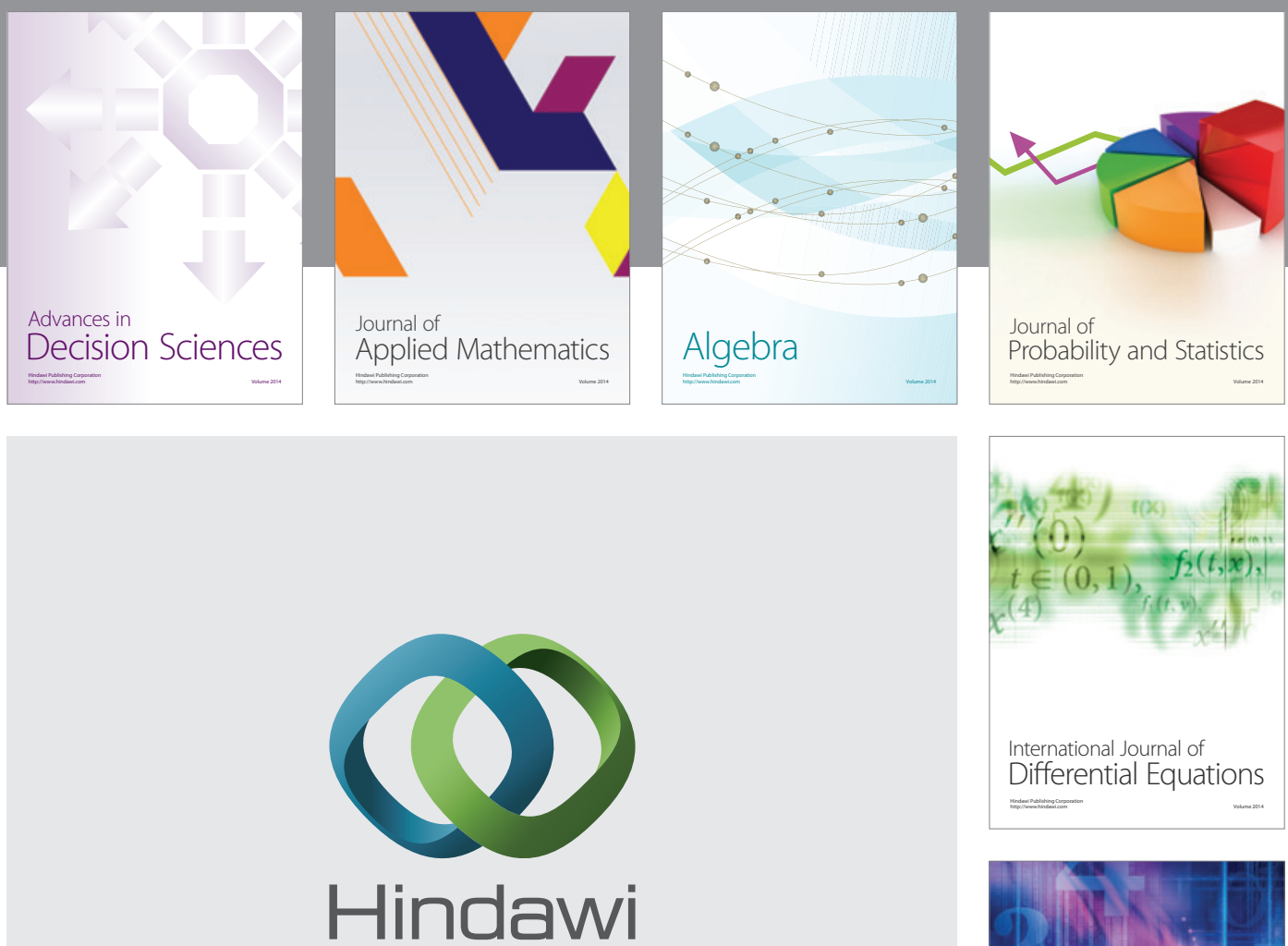

Submit your manuscripts at http://www.hindawi.com
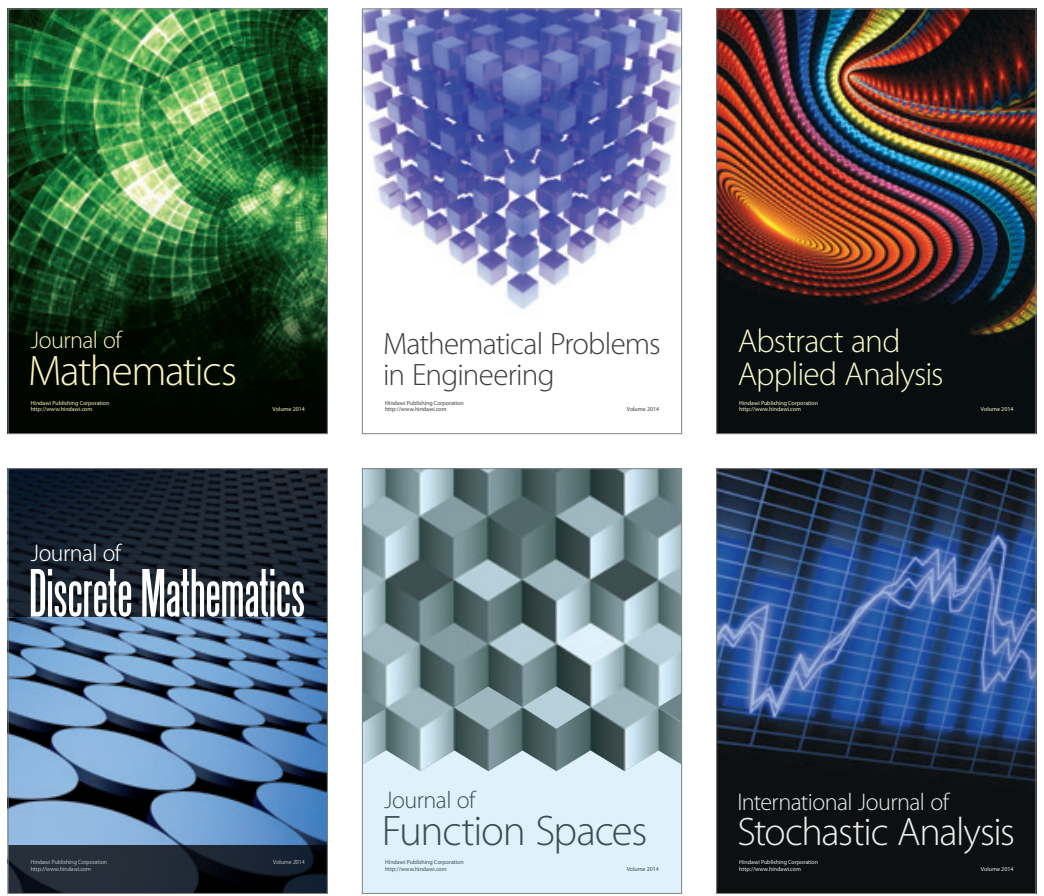

Journal of

Function Spaces

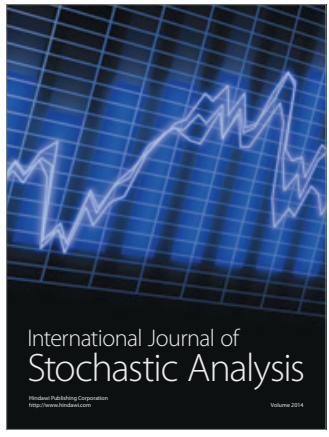

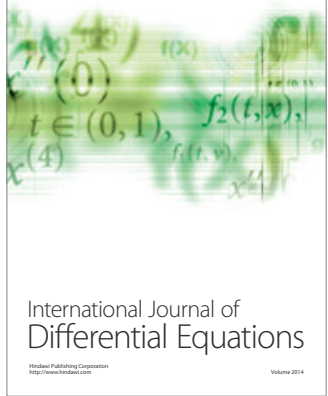
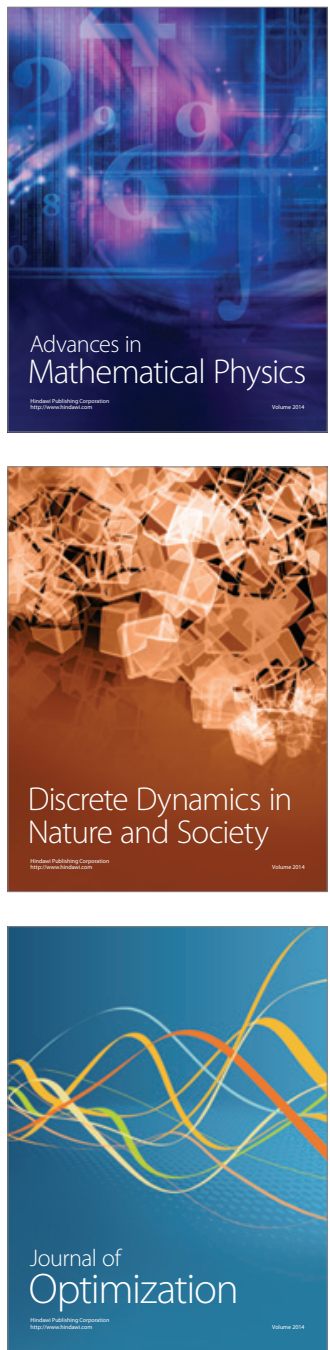